TABLE VIII.-Van den Bergh Reactions

\begin{tabular}{l|c|c|c}
\hline \multirow{2}{*}{ Case } & $\begin{array}{c}\text { Week of } \\
\text { Illness }\end{array}$ & \multicolumn{2}{|c}{ Bilirubin Units per c.cm. } \\
\cline { 3 - 4 } & Direct Reaction & Indirect Reaction \\
\hline M. W. & 6th & 5.8 & 6 \\
J. D. & 8th & 3 & 4 \\
& 9th & 2 & 2 \\
R. L. & 14th & 0 & 0.5 \\
O. G. & 2nd & 18.6 & 19.4 \\
& 8th & 2 & 2 \\
G. J. & 2nd & 41.8 & 44 \\
& 3rd & 16.8 & 18 \\
A. W. & 2nd & 33.8 & 36 \\
29th & 0 & 0.5 \\
& 2nd & 42.4 & 44 \\
& 2nd & 9.6 & 10.4 \\
T. W. R. & 3rd & 15.4 & 4 \\
& 8th & 3r.2 & 1 \\
\hline
\end{tabular}

TABLE IX.--Urine Bilirubin, Urobilinogen, and Urobilin

\begin{tabular}{|c|c|c|c|c|}
\hline Case & $\begin{array}{l}\text { Week of } \\
\text { Illness }\end{array}$ & Bilirubin & Urobilinogen & Urobilin \\
\hline M. W. & $\begin{array}{l}6 \text { th } \\
8 \text { th }\end{array}$ & $\begin{array}{c}\text { Plus } \\
\text { ", }\end{array}$ & $\begin{array}{l}\text { No excess } \\
" \| "\end{array}$ & \\
\hline J. D. & 11 th & Negative & & \\
\hline D. H. J. & 7th & - $U_{r}$ & ic, no urine ava & \\
\hline R. L. & 2nd & Plus & No excess & Negative \\
\hline O. G. & $3 \mathrm{rd}$ & & $" \quad "$ & " \\
\hline A. W. & 3 rd & " & " " & $"$ \\
\hline W. T. & $3 \mathrm{rd}$ & $"$ & $"$ & $"$ \\
\hline T. W. R. & 4th & " & $" \quad "$ & $"$ \\
\hline
\end{tabular}

I have pleasure in acknowledging the ready collaboration of my colleagues, Drs. Esmond Rees and J. Lloyd Davies, and of Mr. Willard Maclean, the clinicians concerned with these patients. My thanks are also given to Mr. Leonard Snow, biochemical technician at the Beck Laboratory.

The detection of nitrous fumes is the subject of a further leaflet issued by the Department of Scientific and Industrial Research ("Methods for the Detection of Toxic Gases in Industry," Leaflet No. 5, H.M. Stationery Office, 3d.). The situations in which nitrous fumes may be encountered in dangerous concentrations include ammonium nitrate works, celluloid works, dyestuffs works, explosives works, nitric acid works, nitro-cellulose paint, lacquer, and leather cloth works, photographic film works, sulphuric acid works (chamber process). They are also encountered in electroplating, engraving, metal cleaning, and photogravure processes, and are formed during oxy-acetylene welding. Nitrous fumes are extremely dangerous on account of their insidious character. There may be, and generally are, no immediate effects, and therefore it is impossible to foretell the serious consequences that may result from inhalation. A workman, unaware that he has inhaled the fumes, continues at work, often remaining well until after he has returned home. Some hours later he becomes restless, with a dry cough and shortness of breath. These symptoms increase, accompanied by a frothy sputum tinged with blood. If appropriate treatment is not applied death follows from oedema of the lungs. Concentrations stronger than 1 in 10,000 are frequently fatal if breathed for more than a few minutes. A concentration dangerous to inhale for even a short time may be hardly noticeable, because no disagreeable symptoms may be produced. Hence any atmosphere in which nitrous fumes are noticeable, either by smell, irritation, or colour, should be regarded as dangerous. The chemical test described in the leaflet is sensitive enough to be readily capable of detecting a concentration of 1 part in 100,000 .

\section{EARLY DIAGNOSIS OF CONGENITAL DISLOCATION OF THE HIP}

BY

\author{
H. A. T. FAIRBANK, D.S.O., M.S., F.R.C.S. \\ Consulting Orthopaedist to King's College Hospital, \\ Consulting Surgeon to the Hospital for Sick \\ Children, Great Ormond Street
}

The purpose of this short paper is to call attention to the fact, not always remembered, that congenital dislocations of the hip can be diagnosed before walking is begun, and to stress the advantages that accrue to the patient from diagnosis at this early age. Unfortunately it is rare for the surgeon to see a case before the age of eighteen months or so, while far too many cases are not diagnosed till much later, when it is quite impossible for treatment, however skilful it be, to produce a result other than one which falls far short of perfection. It can be said without fear of contradiction that the earlier a case is diagnosed the simpler will be the treatment, the greater the chance of a permanent cure, and-a very important point-the more perfect will be the anatomical and functional result. The surgeon is not content with curing the dislocation; he wants to give the patient a hip which will be radiographically normal, or approximately so, and which, therefore, will stand the wear and tear of life like a normal joint.

\section{The Need for Early Recognition}

If the deformity is diagnosed when a child is still below 12 months of age it is not even necessary to do a forcible manipulative reduction under an anaesthetic: simple abduction, increased till extreme, and maintained for a few months, will effect a cure in something like 90 per cent. of the cases. After the age of 12 months reduction and a stable position for the limb can only be achieved by force, the percentage of cures is not so high, and even though the cases are treated before the end of the second year a proportion of the "cures" will show a definite departure from the normal anatomically. It is therefore highly desirable that the diagnosis be made before the child begins to stand and walk. This is not always easy, but it can and should be done. The difficulty of the task is increased by the comparative rarity of the condition in this country. In Northern Italy, where the deformity is relatively common, Putti (1933) has shown what can be achieved by educating practitioners, and even mothers, to keep a constant watch for the signs of an abnormal hip in the early months of life. There is no reason why we should not do something on these lines in this country. The main reason why rare but well-known conditions are not uncommonly missed is not that the diagnosis is necessarily very difficult, but that the correct diagnosis, because of its rarity, never enters the doctor's head.

What may attract a mother's attention to the leg or legs? To quote Putti (1929):

"The mother may have noticed some little asymmetry, something unusual in the shape, the outline, or the attitude of one limb. One limb seems to her shorter than the other; one of the feet turns outward; in separating the lower limbs she saw, or felt, that one went less easily than the other; she observed that one limb was held in a certain degree of flexion or that if she tried to correct this flexion the child cried It is just such small signs that make one suspect a dislocation, and which should induce one to have an $x$-ray examination." 


\section{Signs to Look For}

Now what are the signs the doctor should look for in infants? Asymmetry of the groove between labium and thigh. A slight hollow below the centre of Poupart's ligament. Asymmetry of the creases so commonly seen in the skin and fat on the inner side of the thighs of infants. The gluteal fold may be less distinct than on the normal side. The hips of young infants of both sexes have the slender male contour. If one hip seems more prominent, the contour on this side being that of the female rather than the male, a dislocation should be suspected. Slight shortening of one leg may be noticed as the legs lie parallel and fully extended. If the hips and knees are flexed the knees are not on the same level (Perkins, 1928). One leg may be rolled out rather more than the other. The sex of the patient is of some significance, since, as is well known, congenital dislocation is far more common in females. In about a third of the cases the deformity is bilateral, and in these many of the above suggestive signs will not be apparent. In a bilateral case the breadth of the perineum is increased, and, if the child is not too fat, the thighs will not be in contact. If any of the above signs are present the doctor will naturally proceed to palpation of the hips. In a fat baby, with but a small degree of luxation, it may be difficult or impossible to be sure of displacement at the hip. Nevertheless an attempt should be made to decide the diagnosis by searching with the fingers for those signs which one can make out with ease in an older child.

In a unilateral case the trochanter is raised on the affected side. On palpating the front of the joint the resistance of the head of the femur is not felt. The fingers can be depressed into the joint to a degree impossible on the normal side. This apparent absence of the femoral head from its normal situation must be confirmed by feeling the head elsewhere. The head in congenital dislocations is always to be felt in front of the great trochanter, somewhere between this and the anterior superior spine. On rotating the femur outwards the displaced head becomes more obvious. An abnormal degree of freedom of movement, particularly as regards rotation of the femur, may also be noticed. Abduction, however, may be limited, but this is only marked in older children, particularly in bilateral cases. Telescopic movement may be made out, again more often in the older children, provided always that the child can be induced to relax. When a case is seen only after walking has begun-and this will be later than usual-the suggestive limp and Trendelenburg's sign will be present: both are indicative of instability of the hip, and both are particularly well seen in bilateral dislocations. Lordosis is only marked when both hips are affected.

\section{Differential Diagnosis}

With regard to differential diagnosis, a pathological dislocation is excluded by the history and the absence of a scar. Congenital maldevelopment of the limb without dislocation is excluded by the radiograph: gross deficiency in the formation of the upper part of the femur usually produces a greater degree of asymmetry than is ever seen with congenital dislocation of the hip. Coxa vara is excluded by feeling the femoral head in its correct position in the joint, and failing to feel it in an abnormal position. In every case in which there is the faintest suspicion of a dislocation or other developmental error a radiograph of the two hips should be taken without delay. Particular trouble should be taken to ensure that the two legs are in identical standard positions when the film is being exposed, while the " reading" of the film of a young infant calls for special care.

The radiographic signs in an infant are: (1) shelving of the upper portion of the acetabulum; (2) diminution in size of the ossific centre for the head, if these centres are visible at all ; and (3) displacement of the femur outwards and upwards. The first is invariably present; the second naturally depends on the age of the child, and is of no help in a bilateral case, but is invaluable in a unilateral case when the heads are visible; the third may be definite and conclusive, but is not by any means always so. In a tiny baby with a minimal degree of displacement, particularly in the bilateral case, a definite decision on the point may be impossible. The condition in which the roof of the socket is shelving rather more than that on the opposite side, and the displacement of the femur is minimal or absent, has been termed a "pre-dislocation." It is wiser to treat such a case as a dislocation rather than run the risk of the imperfection of the joint being rediscovered in middle life when arthritic changes have developed in a subluxated hip.

In conclusion, I suggest that an effort should be made to get these cases under treatment at an earlier age than has been usual in the past by constantly looking for them in child welfare centres and elsewhere, and by sending to the radiologist every suspicious case. Once the diagnosis is made, the sooner the case is sent to an orthopaedic clinic the better.

REFERENCES

Perkins, G. (1928). Lancet, 1, 648.

Putti, V. (1929), J. Bone Jt. Surg., 11, 798. (1933). Ibid., 15, 16.

\section{PNEUMOCOCCAL PNEUMONIA, COMPLI- CATING PULMONARY TUBERCULOSIS, TREATED WITH M \& B 693}

\author{
BY \\ J. H. CRAWFORD, M.D. \\ Assistant Medical Director, Preston Hall Sanatorium, \\ Aylesford, Kent
}

In view of the recent interest aroused in the treatment of pneumococcal infections with the preparation M \& B 693, the following notes of a patient with pulmonary tuberculosis who developed pneumococcal pneumonia and was treated with this drug may prove of some value.

\section{Case Record}

The patient was a man aged 22 who had suffered from pulmonary tuberculosis for three years. On admission to the sanatorium he was found to be suffering from bilateral disease, radiography of the chest revealing extensive fibro-caseous disease over the upper and mid zones on the right side, with scattered infiltration oyer the left lung and cavitation below the clavicle on this side. The sputum was heavily positive, and the sedimentation rate at the end of one hour $(50 \mathrm{~mm}$. tube) was 18 .

Under routine treatment he made fair progress until the beginning of July, 1938, when he appeared to have contracted a chill. His temperature rose to $103^{\circ} \mathrm{F}$., and next day physical examination revealed signs of consolidation of the right base. He had become dyspnoeic and his cough was increasingly troublesome. On the following day signs of a pleural effusion were found on the right side, and $18 \mathrm{oz}$. of greenish-yellow pus were aspirated. At this stage the leucocyte count was 12,400 per c.mm. and the sedimentation rate had increased to 28 , a graph plotted at five-minute intervals showing a very rapid fall in the first half-hour. The 